\title{
FUNCTIONS ON MULTIPLIERS OF $p$-FOURIER ALGEBRAS
}

\author{
BY MICHAEL J. FISHER ${ }^{1}$
}

Communicated by R. Seeley, January 22, 1973

I. Introduction. Let $G$ denote a locally compact abelian (LCA) group and let $\Gamma$ denote the group which is dual to $G$. If $1 \leqq p \leqq \infty$, let $L_{p}(G)$ (or $L_{p}(\Gamma)$ ) denote the space of $p$-power integrable functions with respect to Haar measure on $G$ (or on $\Gamma$ ); let $C(G)$ denote the algebra of bounded continuous functions on $G$ and let $C_{0}(G)$ consist of those functions in $C(G)$ which vanish at infinity. In [2] and [3] Figa-Talamanca and FigaTalamanca and Gaudry studied the $p$-Fourier algebra

$$
A_{p}(\Gamma)=\left[L_{p}(\Gamma) \hat{\otimes} L_{p^{\prime}}(\Gamma)\right] / K
$$

where $1 \leqq p<\infty, p^{\prime}$ is conjugate to $p, \hat{\otimes}$ is the projective tensor product, and $K$ denotes the kernel of the convolution operator $c: L_{p} \otimes L_{p^{\prime}} \rightarrow C_{0}(\Gamma)$ by $c(f \otimes g)(y)=f * g(y) . A_{p}(\Gamma)$ carries the quotient norm. Herz [7] showed that $A_{p}(\Gamma)$ is a Banach algebra under pointwise multiplication. In [2] Figa-Talamanca showed that the dual space of $A_{p}(\Gamma)$ is isometrically isomorphic to the space $M_{p}(\Gamma)$ of bounded, translation invariant, linear operators on $L_{p}(\Gamma)$ and that the weak operator topology on $M_{p}(\Gamma)$ and the weak*-topology agree on bounded sets. Implicit in [2] is the fact that $A_{2}(\Gamma)$ is isometrically isomorphic with $A(\Gamma)$, the algebra of Fourier transforms of integrable functions on $G ; A(\Gamma)$ is equipped with the inherited norm; see also [1]. Hewitt's factorization theorem is used in [3] to prove that $A_{1}(\Gamma)$ is $C_{0}(\Gamma)(C(\Gamma)$ when $\Gamma$ is compact).

Let $B_{p}(\Gamma)$ denote the algebra of functions $f$ in $C(\Gamma)$ which satisfy: $f(\gamma) h(\gamma) \in A_{p}(\Gamma)$ whenever $h \in A_{p}(\Gamma) . B_{p}(\Gamma)$ is a commutative and semisimple Banach algebra under pointwise addition and multiplication when it is equipped with the operator norm; $B_{p}(\Gamma)$ is the algebra of bounded multipliers on $A_{p}(\Gamma)$. If $1<p<\infty$ and if $p^{\prime}$ denotes the index conjugate to $p$, then $A_{p^{\prime}}=A_{p}$ and $B_{p^{\prime}}=B_{p}$, so that we may restrict $p$ to $1<p<2$. It is easy to see that $B_{1}(\Gamma)=C(\Gamma)$, and Helson's theorem $\left[11\right.$, p. 73] says that $B_{2}(\Gamma)$ is the algebra of Fourier transforms of bounded measures on $G$ with the inherited norm. Since the inclusions $A_{2}(\Gamma) \subset$ $A_{p}(\Gamma) \subset A_{1}(\Gamma)$ are continuous if $1<p<2$, it follows that the maximal

AMS (MOS) subject classifications (1970). Primary 43A22; Secondary 46L20.

Key words and phrases. Functions of Gel'fand transforms, multipliers on $L_{\boldsymbol{r}}, p$-Fourier algebra, multipliers of Fourier algebras, isomorphisms of Fourier algebras.

${ }^{1}$ Research supported in part by NSF grant GP-24574. 
ideal space of $A_{p}(\Gamma)$ can be identified with $\Gamma$. Thus $B_{p}(\Gamma)$ is an algebra of multipliers in the sense of Larsen [9]; we refer to this monograph for the basic facts regarding multiplier algebras.

The purpose of this note is to show that the only complex valued functions of a complex variable which operate on (the Gel'fand transforms of) $B_{p}(\Gamma)$ are entire functions when $\Gamma$ is not compact. If $\Gamma$ is compact, $A_{p}(\Gamma)=B_{p}(\Gamma)$ and the class of functions which operates on $B_{p}(\Gamma)$ is less restrictive. When $\Gamma$ is not compact, this will imply that the algebra $B_{p}(\Gamma)$ is not selfadjoint and not regular and that $\Gamma$ is not dense in the maximal ideal space of $B_{p}(\Gamma)$. The basic result (Theorem 1, below) from which this information follows can also be used to describe the isometric isomorphisms of $A_{p}(\Gamma)$ onto $A_{p}(\Lambda)$ when $\Lambda$ is a second LCA group. A detailed development of these topics will be given in [5].

II. Functions operating on multipliers. In [6] Hahn proved that if $1 \leqq p \leqq 2$ and if $f \in L_{p}(\Gamma)$ and $g \in L_{p},(\Gamma)$, then $h(y)=f * g(y)$ is the Fourier transform of an operator $T_{h}$ in $M_{r}(G)$ if $\left|1 / r-\frac{1}{2}\right| \leqq|1 / p-1|$ for which $\left\|T_{h}\right\|_{r} \leqq\|f\|_{p}\|g\|_{p^{\prime}}$. We have the following extension of this result.

THEOREM 1 . Let $1 \leqq p \leqq 2$ and let $\left|1 / r-\frac{1}{2}\right| \leqq|1 / p-1|$. Then $f$ in $B_{p}(\Gamma)$ is the Fourier transform of an operator $T_{f}$ in $M_{r}(G)$ and the map $f \rightarrow T_{f}$ faithful, norm decreasing representation of $B_{p}(\Gamma)$ as an algebra of bounded, translation invariant operators on $L_{r}(G)$.

It is clear that Hahn's map $h \rightarrow T_{h}$ extends to a norm decreasing isomorphism of $A_{p}(\Gamma)$ into $M_{r}(G)$. Now Hahn's Lemma 1 and the fact that $A_{p}(\Gamma)$ has a bounded approximate identity (since $A_{2}(\Gamma)$ does) can be used to extend $h \rightarrow T_{h}$ to all of $B_{p}(\Gamma)$.

The Fourier transforms of bounded measures on $G$ are elements of $B_{p}(\Gamma)$; denote this subalgebra by $B_{2}(\Gamma)$.

THEOREM 2. Suppose that $\Gamma$ is not compact and that $1<p<2$. Let $F$ be a complex valued function defined on $[-1,1]$ for which $F(\hat{\mu}(\gamma)) \in B_{p}(\Gamma)$ for every $\hat{\mu} \in B_{2}(\Gamma)$ with range in $[-1,1]$. Then $F$ admits an extension to all of $\boldsymbol{C}$ as an entire function.

This follows from Igari's Theorem 1 of [8] and from Theorem 1 above. When $\Gamma$ is compact but not discrete, $F$ must be analytic in a neighborhood of $[-1,1]$.

Now, by reasoning as in $[\mathbf{1 1}$, Chapter 6] or as in [8], one may conclude that

THEOREM 3. Suppose that $\Gamma$ is noncompact and that $1<p<2$. Then:

1 . If $F$ is a complex valued function of a complex variable for which $F(\hat{f})$ is the Gel'fand transform of a function in $B_{p}(\Gamma)$ whenever $\hat{f}$ is, then $F$ is an entire function. 
2. For any complex number $z$ there is a real valued function $f$ in $B_{p}(\Gamma)$ and a complex homomorphism $h$ of $B_{p}(\Gamma)$ for which $h(f)=z$.

3. $B_{p}(\Gamma)$ is not selfadjoint and not regular.

4. $\Gamma$ is not dense in the maximal ideal space of $B_{p}(\Gamma)$.

5. There is a function $f$ in $B_{2}(\Gamma) \subset B_{p}(\Gamma)$ with $f \geqq 1$ on $\Gamma$ for which $f^{-1}$ is not in $B_{p}(\Gamma)$.

The analogy between $B_{p}(\Gamma)$ and $B_{2}(\Gamma)$ does not end here, but we shall wait to describe the situation more completely in [5].

III. Isomorphism. Let $\Gamma$ and $\Lambda$ be LCA groups and let $A_{p}(\Gamma)$ and $A_{p}(\Lambda)$ denote their respective $p$-Fourier algebras for $1<p<2$. It follows from Theorem 1 of $\S$ II and from a theorem of Strichartz [12] that the only isometric multipliers on $A_{p}(\Gamma)$ are complex unit multiples of characters on $\Gamma$. This is the basic fact needed to prove

THEOREM 4. If $\Phi$ is an isometric isomorphism of $A_{p}(\Gamma)$ onto $A_{p}(\Lambda)$, then there is a topological isomorphism $\alpha$ of $\Lambda$ onto $\Gamma$ and an element $\gamma_{0}$ of $\Gamma$ such that $\Phi(h)(\lambda)=h\left(\gamma_{0}+\alpha(\lambda)\right)$.

Corollary 4.1. $\Gamma$ is topologically isomorphic to $\Lambda$ if and only if $A_{p}(\Gamma)$ is isometrically isomorphic to $A_{p}(\Lambda)$; i.e. $A_{p}(\Gamma)$ determines $\Gamma$.

The proof relies on the facts that $\Phi$ extends to $B_{p}(\Gamma)$, that $\Phi$ maps isometric multipliers to isometric multipliers, and that $G$ is topologically isomorphic to the multipliers $\left\{g_{0}(\gamma) \mid g_{0} \in G\right\}$ when this group is equipped with the strong operator topology.

$A_{p}(\Gamma), B_{p}(\Gamma)$, and $M_{p}(\Gamma)$ forms an interrelated system of algebras which we first studied in [4]. There, we proved Theorem 1 of $\S I I$ in the context of a continuity theorem of Lévy type for $M_{r}(G)$. No applications of Theorem 1 were given in [4].

\section{REFERENCES}

1. P. Eymard, Lalgébra de Fourier d'un groupe localment compact. Bull. Soc. Math. France 92 (1964), 181-236. MR 37 \#4208.

2. A. Figa-Talamanca, Translation invariant operators on $L_{p}$, Duke Math. J. 32 (1965), 495-501. MR 31 \#6095.

3. A. Figa-Talamanca and G. I. Gaudry, Density and representation theorems for multipliers of type $(p, q)$, J. Austral. Math. Soc. 7 (1967), 1-6. MR 35 \#666.

4. M. J. Fisher, Recognition and limit theorems for $L_{p}$-multipliers, Studia Math. 50 (to appear).

5. - Multipliers and p-Fourier algebras, Studia Math. (to appear).

6. L.-S. Hahn, On multipliers of p-integrable functions, Trans. Amer. Math. Soc. 128 (1967), 321-335. MR 35 \#4677.

7. C. Herz, The theory of p-spaces with an application to convolution operators, Trans. Amer. Math. Soc. 154 (1971), 69-82. MR 42 \#7833. 
8. S. Igari, Functions of $L_{p}$-multipliers, Tôhoku Math. J. 21 (1969), 304-320.

9. R. Larsen, The multiplier problem, Lecture Notes in Math., vol. 105, Springer-Verlag, Berlin, 1969.

10. L. H. Loomis, An introduction to abstract harmonic analysis, Van Nostrand, Princeton, N.J., 1953. MR 14, 883.

11. W. Rudin, Fourier analysis on groups, Interscience Tracts in Pure and Appl. Math., no. 12, Interscience, New York, 1962. MR 27 \# 2808.

12. R. Strichartz, Isomorphisms of group algebras, Proc. Amer. Math. Soc. 17 (1966), 858-862. MR 33 \#1751.

Department of Mathematics, University of Montana, Missoula, Montana 59801 